\title{
COMMUNICATED AND NON-COMMUNICATED ACTS IN RELEVANCE THEORY
}

\author{
Steve Nicolle
}

\begin{abstract}
According to relevance theory (Sperber and Wilson 1986; Blakemore 1991) some cases of communication depend on the hearer recognising that a particular speech act, for example admitting, betting or promising, is being performed. These are 'communicated' acts. Other cases of communication do not depend on the hearer recognising that a particular speech act, for example predicting, warning or permitting, is being performed. These are 'non-communicated' acts. In the case of non-communicated acts communication is successful so long as the hearer recovers adequate contextual effects without having to recognise the speaker's intentions. Against this view, I will argue that each of the speech acts considered to be noncommunicated in the relevance theory literature fall into one of two categories. The speech acts in one category contribute to the strength of associated assumptions, and those in the other convey socially relevant information. I will argue that according to relevance theory both types of speech act must be recognised and that they are in fact communicated. If relevance theory is to be internally consistent, therefore, the distinction between communicated and non-communicated speech acts must be abandoned.
\end{abstract}

Keywords: Relevance theory, Communication, Non-communication, Speech act, Performative

\section{Introduction}

Some of the distinctions posited by relevance theory, such as those between explicature and implicature, conceptual and procedural information, and descriptive and interpretive uses of language, seem to be theoretically well-founded and descriptively useful. They have been refined and their implications explored in various areas of language use, philosophy and cognition (see for example the volumes edited by Carston and Uchida 1998; Rouchota and Jucker 1998; Andersen and Fretheim in press). At a theoretical level, these distinctions have proved capable of highly productive synthesis; for example, Wilson and Sperber (1993) discusses the contribution of conceptual and procedural information to the recovery of explicatures and implicatures, whilst Nicolle (in press) relates the distinction between conceptual and procedural information to descriptive and interpretive uses of particles in two African languages. If these distinctions are in fact well-founded, it is only to be expected that they will contribute to the development of relevance theory and shed light on natural language data.

However another theoretical distinction proposed in relevance theory, the distinction between communicated and non-communicated speech acts, has so far proved to be far less productive, both theoretically and descriptively. Theoretically, this distinction 
has failed to make any significant contribution to the development of relevance theory, and descriptively it has had little impact on the study of speech acts. I will argue that the distinction between communicated and non-communicated speech acts violates some basic principles of relevance theory and runs counter to recent developments in the study of social interaction from the perspective of relevance theory (e.g. Escandell-Vidal 1996; Jary $1998 \mathrm{a}, \mathrm{b})$. It is not surprising, therefore, that it has failed to make a significant contribution to the development of relevance theoretic pragmatics.

The main thrust of my argument will be that all (non-institutional) speech acts are communicated acts. All of the speech acts which have been characterised as noncommunicated in the relevance theory literature concern either (i) the strength with which an assumption is held or (ii) socially relevant information. In the first case, I will argue that acts such as predicting, claiming or hypothesising, which indicate the strength with which an assumption is held (that is, the degree of speaker commitment to a proposition), must be identified in order for a hearer to determine the intended contextual effects of an utterance. In the second case, acts which indicate socially relevant information are always worth recovering since all information pertaining to social relations is highly relevant (in the relevance theory sense). Since the identification of all such acts is essential for the comprehension of the utterances through which they are performed, I conclude that they are communicated acts.

In the following section I will introduce the distinction between communicated and non-communicated acts. Then in sections 3 and $4 \mathrm{I}$ will discuss acts which indicate the strength of assumptions and acts which convey socially relevant information respectively. In section $5 \mathrm{I}$ will briefly discuss the role of performative verbs before concluding in section 6 .

\section{Communicated and non-communicated acts}

According to Sperber and Wilson (1986: 244-6) and Blakemore (1991; 1992: 91-94), some cases of communication depend on the hearer recognising that a particular speech act, for example admitting, betting, promising or thanking, is being performed. These are communicated acts. Other cases of communication do not depend on the hearer recognising that a particular speech act, for example predicting, warning, suggesting or permitting, is being performed. These are non-communicated acts. In the case of non-communicated acts, communication is deemed successful so long as the hearer recovers adequate contextual effects from the utterance apart from recovering the speaker's intentions. So, in (1) and (2) communication is deemed successful so long as adequate contextual effects can be derived from processing the information that the weather will be warmer on the day following the utterance or that the path is slippery in the place indicated; it is not necessary for the hearer to understand that the speaker is predicting in (1) or warning in (2).

(1) The weather will be warmer tomorrow. (Sperber and Wilson 1986: 245)

(2) The path is slippery here. (Blakemore 1991: 201)

The relevance theory definition of a communicated act is stated purely from the hearer's perspective as an act whose identification is essential to the comprehension of an 
utterance. An act can also be described as communicated from the speaker's perspective; that is, it is communicated in the sense of being an act which the speaker intends the hearer to recover. My argument will primarily be against the existence of non-communicated acts in the relevance theory sense, but I will also demonstrate that if an act is relevance theorycommunicated it is also speaker-communicated.

The relevance theory approach is at odds with the central assumption of speech act theory, ${ }^{1}$ namely that successful communication requires that a hearer identify the speech act being performed by a speaker. As Blakemore (1991: 199) observes, if communication depends on the recognition of intentions to perform particular speech acts, then utterances which do not depend on being recognised as performing particular acts in order to be performed successfully are not acts of communication at all. With respect to institutional acts such as naming ships and baptising babies, this argument does not undermine speech act theory per se, rather it demonstrates that certain utterances previously dealt with by speech act theorists are not in fact communicated acts. Thus far I see no reason to disagree with the relevance theory view concerning institutional acts, since naming ships and baptising babies can be successfully performed when an audience fails to recognise the speaker's intentions or even when there is no audience.

Let us turn to utterances which constitute genuine acts of communication. Other institutional acts, that is, acts which can only be interpreted within the framework of a particular social institution, can be communicated. These include bidding at trumps and promising (Sperber and Wilson 1986: 244-5; Blakemore 1991: 200). Sperber and Wilson claim that the study of such acts belongs to the study of institutions and not to pragmatics. Because my aim in this article is to argue against the notion of speech acts which are both non-institutional and non-communicated (henceforth 'non-communicated acts'), rather than to debate the scope of pragmatics, I will not discuss this claim further here. However, the arguments proposed in section 3, dealing with speech acts and socially relevant information, would suggest that even institutional acts can be part of pragmatics if they are communicated.

Just as institutional acts can be either communicated or non-communicated, so, the relevance theory literature claims, can non-institutional acts. Cases of non-institutional communication in which the hearer must recognise that a particular speech act is being performed in order for communication to be successful include conceding and admitting. Blakemore (1991: 200) characterises admitting as a communicated act in the following way:

"Consider a situation in which I, after strenuously denying your accusation that I watch a certain soap opera on television, eventually give up and produce the utterance in (3).

(3) OK. I watch it.

Obviously, since you already believe that I watch the programme, the proposition in (4) cannot be relevant to you. The main relevance of the

\footnotetext{
${ }^{1}$ For a detailed analysis of some differences between speech act theory and relevance theory, see Dominicy and Franken (forthcoming).
} 
utterance must lie in the proposition in (5).

(4) The speaker of (3) watches Neighbours.

(5) The speaker of (3) is admitting that she watches Neighbours."

In contrast to non-institutional acts which are communicated, others are viewed as being non-communicated. Sperber and Wilson (1986: 245) claim that (1) could be a prediction without the hearer having to recover the information in (6):

(1) The weather will be warmer tomorrow.

(6) The speaker of (1) is predicting that the weather will be warmer tomorrow.

They are not claiming that the proposition in (6) cannot be recovered, merely that the recovery of the proposition in (6) is not an essential element in the interpretation of an utterance of (1). In contrast, the recovery of the proposition in (5) is essential if an utterance of (3) is to be interpreted correctly.

Similarly, Blakemore (1991: 201) argues that (2) could be interpreted as a warning without the hearer having to recover the proposition in (7):

(2) The path is slippery here.

(7) The speaker of (2) is warning the hearer that the path is slippery here.

Blakemore argues that the main relevance ${ }^{2}$ of (2) lies just in the proposition that the path is slippery in the place indicated. This is because the contextual implications that the speaker intended the hearer to recover, for example that the hearer might slip and hurt himself in the place indicated, are derivable from the proposition in (2) without the hearer having to recover the proposition in (7). The speech act of warning is therefore not a communicated act, since it is not essential for the comprehension of the utterance in (2).

This, then, is the distinction made between communicated and non-communicated acts: Non-institutional speech acts whose identification is essential to the comprehension of the utterances which convey them are communicated acts, and those which it is not essential to recover are non-communicated acts. In the following sections I will discuss speech acts such as predicting in (1) and warning in (2) and argue, for different reasons in each case, that these are communicated acts.

${ }^{1}$ I follow Franken (1997) in regarding Blakemore's use of the term 'main relevance' as referring to those communicated hypotheses which produce the greatest number of contextual effects. In talking sometimes of the main relevance of an utterance and at other times just of the relevance of an utterance (e.g. ibid. 202, 204, 206), Blakemore brings ambiguity into the term 'communicated'. If the main relevance of an utterance lies in the proposition expressed rather than in any higher-level information, it is still possible for the higher-level information to have secondary relevance. That is, recovery of the proposition expressed is essential for successful communication whereas recovery of higher-level information in some sense optional. However, if the relevance of an utterance lies solely in the proposition expressed, then the higher level information is irrelevant. Blakemore seems to favour the first option, and to be using the term 'relevance' sometimes as a short form of 'main relevance'. This fits with Sperber and Wilson's definition of a communicated act as an act whose identification is essential to the comprehension of an utterance. Rather than adopt Franken's (1997) proposal and use the term 'communicational', I will continue to refer to 'communicated' acts, but in the sense of acts whose identification is essential to comprehension. 


\section{Speech acts and the strength of assumptions}

Against the position outlined above, I will argue that predicting in (1), for instance, must be a communicated act given the basic types of contextual effect posited in relevance theory. According to Sperber and Wilson (1986: 108) a deduction based on the union of new information and old information may give rise to contextual effects. During a conversation, for example, new information will be derived from a proposition expressed by means of an utterance, and old information might consist of assumptions derived from previous utterances or entertained prior to the conversation. Together, old information and new information constitute the premises and the resulting contextual effects constitute the conclusion to an inferential deduction.

Contextual effects come in three kinds: Contextual implications, strengthening of existing assumptions, and contradiction and elimination of existing assumptions. A crucial factor in deriving all three types of contextual effect is the strength of the assumptions. By the strength of an assumption I mean the degree to which the hearer is committed to the truth of an assumption that constitutes one of the premises in a deduction. Concerning contextual implications, Sperber and Wilson (1986: 112) write:

consider first how contextual implication, our original example of a contextual effect, fares when the strength of assumptions is taken into account. Contextual implication is a sub-type of synthetic implication. Consider, then, the relation between a synthetic implication and the premises effectively used in deriving it. [...] each premise is an argument for the conclusion, or, as we propose to say, each of the premises effectively used in the derivation of a synthetic implication strengthens the conclusion which they jointly imply. The contribution of each individual premise to the strength of this joint conclusion is a function of its own degree of strength.

The strength of the assumptions functioning as premises in the other two types of contextual effect - strengthening and contradiction of existing assumptions - is also clearly important. The degree to which new information strengthens an existing assumption depends on the strength of the new information. Similarly, new information can only eliminate an existing and contradictory assumption if the strength of the new information is greater than the strength of the existing assumption, that is, if the hearer believes that the likelihood of the new information being true is greater than the likelihood of the old information being true.

Knowing whether an utterance of (1) is a prediction, hearsay, a meteorological forecast, or a guess will have a bearing on whether an utterance of (1) succeeds in strengthening or contradicting an existing assumption, or whether it gives rise to a strongly or weakly held contextual implication. But is the recognition of speech acts such as predicting therefore a necessary condition for the recovery of contextual effects? That is, is the identification of such acts essential to the comprehension of an utterance, such that these speech acts are, by definition, communicated? Consider a situation in which a piece of information conveyed by an utterance is new to the hearer. How is the hearer to determine its strength? If the hearer assumes that the speaker is being truthful then he will need to assess both the speaker's commitment to the proposition expressed and the credibilty of the speaker. (This assessment will be constrained by the principle of relevance, 
which requires that the assumptions made manifest by the utterance are relevant enough to be worth processing. Thus, in some cases the principle of relevance will impose a lower limit on the hearer's assessment of the speaker's committment to the proposition expressed, below which any resulting contextual effects are not worth processing.) Assessment of the strength of the speaker's commitment to the proposition expressed by her utterance requires that the hearer identify the associated speech act: Predicting, reporting hearsay, making a meteorological forecast, guessing, etc. Identifying the associated speech act is therefore an essential part of the utterance interpretation process in such cases and the act is by definition a communiated act.

Since the utterance interpretation process requires that such speech acts be identified, and the principle of relevance requires that each utterance is the most relevant one that the speaker could have used (given her abilities and preferences), a speaker's utterance should be structured in such a way that it will enable the hearer to identify the associated speech act. Speech acts such as predicting, claiming or hypothesising, which indicate the strength of an assumption, therefore constitute an essential part of what a speaker would wish to communicate by her utterance and they are therefore communicated not only in the relevance theory sense that their identification is essential to the comprehension of an utterance but also in the sense of being speaker-intended.

The above argument covers cases where the hearer believes that the speaker is being truthful and where the information provided by the speaker is new to the hearer. However, what happens in cases where the speaker is lying or where the hearer knows that the new information provided by the speaker is false ${ }^{3}$ (or where the hearer knows that the information is definitely true whereas the speaker is not totally committed to its truth)? If the speaker is lying, her intention will be to mislead the hearer, that is, to get the hearer to process the false information as if it were true or probably true and hence to derive certain contextual effects as a result. Since the speaker in this case is simulating a genuine exchange of information, she will communicate a proposition and associated speech act as in the case discussed above. If the lie is to be successful, the hearer must fail to make an adequate assessment of the speaker's credibility with the result that the strength with which he comes to entertain the communicated assumption fails to correspond to reality. In the case where the hearer knows that the information provided by the speaker is false (or that the information is definitely true whereas the speaker is not totally committed to its truth), the speaker, whether lying or attempting to be truthful, will not know that this is the case. (If a speaker tells a hearer something that both know to be false the communication will be understood as a case of irony, a joke, fiction, etc.; speech acts such as predicting play no role in such cases.) If the speaker is unaware that the information she is providing is false, then from her perspective this case is the same as that discussed initially; as far as the speaker is concerned, the speech act which indicates the strength of her assumption constitutes an essential part of what the hearer must recover in order to understand her utterance correctly, and it is therefore a communicated act.

\footnotetext{
${ }^{3}$ I am grateful to an anonymous referee for bringing this particular case to my attention.
} 


\section{Speech acts and socially relevant information}

According to Blakemore (1991: 209) begging, requesting, advising and permitting are noncommunicated acts but ordering is a communicated act because it is institutional; an utterance only counts as an order if the speaker is exercising authority over the hearer which is legitimised by some social institution. This division of speech acts into communicated and non-communicated on the basis of whether they are institutional or noninstitutional seems arbitrary. Although a speaker may be legitimately allowed to issue an order by virtue of the power relations holding between the speaker and the hearer, that is, according to some institutional criteria, she is not obliged to perform the speech act of ordering rather than, say, requesting. Just as the choice of honorific or non-honorific forms in Japanese helps to create a context with respect to "preferred interpersonal relations and identities" which can take precedence over institutional relations and identities (Okamoto 1999: 70; see also Arundale 1999: 125), so choosing to perform a particular speech act, such as ordering or requesting, serves to reinforce or modify the social environment of the interlocutors. So long as this social environment or context consists of mutually manifest assumptions, relevance theory is capable, in principle, of providing an account of such socially motivated uses of language (see for example Escandell-Vidal 1996; Jary 1998a; Terkourafi 1999).

I consider not only begging, requesting, advising and permitting, but all such 'socially relevant' speech acts (that is, acts which both depend on and affect the social environment of the interlocutors) to be communicated acts. Take for example the act of warning in (2) (repeated below):

The path is slippery here.

Whilst I agree with Blakemore that the proposition that the path is slippery near the speaker leads to contextual implications (for example that the hearer might slip and hurt himself in the place indicated), the fact that (2) might be intended as a warning has obvious social consequences. When the speaker warns the hearer that the path is slippery, the speaker is acting altruistically and the hearer is thereby placed in the debt of the speaker. Failure to respond appropriately, for example by thanking the speaker, has the potential to impair the social relationship between speaker and hearer. It seems that in such situations hearers cannot help but recognise the intention to warn and, in cultures where it is appropriate, the offering of thanks happens virtually automatically.

I will argue, with Jary (1998b), that information relating to an individual's social environment is inherently relevant, and furthermore that within the framework of relevance theory the recovery of information relating to social relations is an essential element of the comprehension process. When the recovery of such information depends on the identification of a particular speech act, that speech act is by definition a communicated act.

Jary (1998b: 165), following Humphrey (1976) and Tooby and Cosmides (Tooby and Cosmides 1992; Cosmides and Tooby 1992), suggests that information about an individual's social environment is easy to process, since the human cognitive system is designed to be able "to form and maintain alliances, to keep track of one's relative status and that of others, to calculate the likely effect of one's actions on the way others think of you and their likely responses to your actions." Since we live in complex social groupings, 
such information typically yields adequate contextual effects. Information that is both easy to process and likely to yield adequate contextual effects is, in relevance theory, relevant information:

If it is accepted that a proper function of human cognition is to provide an adequate representation of the individual's social environment then, on [relevance theory] terms, it follows that stimuli which make manifest assumptions relating to this aspect of her cognitive environment will be highly relevant as the assumptions they give rise to will be easy to process, this being what the cognitive system - or rather, a significant part of it - has been designed for. (Jary 1998b: 166) ${ }^{4}$

A major source of socially relevant information comes from the ostensive behaviour of other people, that is, "behaviour which makes manifest an intention to make something manifest" (Sperber and Wilson 1986: 49), particularly acts of linguistic communication. If an utterance conveys information about the social environment of the speaker and the hearer, and considerations of relevance indicate that such information is worth processing, then such information will be processed. Failure to process such information may not impair the recovery of contextual implications derivable from the proposition expressed, but it may adversely effect the maintenance of social relations between the interlocutors. The identification of speech acts such as warning, begging, and permitting, which convey socially relevant information, is therefore an essential element in utterance comprehension. Since the definition of a communicated act provided in the relevance theory literature (Sperber and Wilson 1986: 245) is of a speech act whose identification is essential to the comprehension of an utterance, these socially relevant speech acts are therefore communicated acts.

Such speech acts are also communicated in the sense of being speaker-intended. Socially relevant information can be obtained from other aspects of linguistic communication; for example a person's accent or the language they use may provide an indication of their social class or ethnic identity. However, such sources of information are often beyond a speaker's control, and therefore cannot be communicated in the sense of intended by the speaker. ${ }^{5}$ The difference with speech acts is that speakers are always able to exercise a choice in regard to which speech acts to perform. I may or may not be able to choose which language to address somebody in, but I always have the choice to order, request or beg, to warn or advise or permit.

So, not all socially relevant information is communicated, but it is typically easy to process and yields adequate contextual effects (that is, modifications to a certain part of the hearer's cognitive environment). A rational speaker (and relevance theory assumes that interlocutors behave rationally) will therefore take this into account when producing her utterance. In interpreting any utterance, a hearer will inevitably attempt to identify the

\footnotetext{
${ }^{4}$ The question of whether the part of the cognitive system which deals with the processing of socially relevant information should be understood as a module or combination of modules, or as a function of the central system, falls beyond the scope of this discussion. For a general consideration of modularity, see for example Sperber (1994).

${ }^{5}$ However, when a speaker is able to choose the language she wishes to use, this choice may play a definite social and communicative role. For example, according to Myers-Scotton's "markedness model" of code-switching (Myers-Scotton 1993; 1995), a speaker's choice of a particular language or mixture of languages on a given occasion may convey information relating to presentation of self and the negotiation of interpersonal relations.
} 
speech act being performed; either it will help the hearer to determine the strength of the associated proposition (see above) or it will yield socially relevant information. In the second case the utterance conveys the expectation that the effort required to identify the associated speech act will typically be small but will yield adequate contextual effects: That is, it brings with it an expectation of relevance. Since a speaker has a choice as to whether or not to perform a particular speech act, a rational speaker will produce the utterance which is most likely to lead the hearer to recover the information that the speaker wants him to recover. Speech acts such as warning, begging, permitting requesting and advising should therefore be viewed as communicated acts from the speaker's perspective also.

\section{Performatives and the strength of assumptions}

The position of performative expressions deserves consideration whether we accept that all speech acts are communicated, as I have argued, or that some acts are communicated and others are non-communicated, following Sperber and Wilson (1986) and Blakemore (1991).

According to Blakemore (1991: 202-7, 1992: 95-100) performatives describing noncommunicated acts express propositions but do not contribute to the truth-conditions of the utterances that contain them. For example, the performative in (8) expresses the higherlevel proposition in (9) but the truth of an utterance of (8) lies in the proposition in (10). Blakemore argues that in general a hearer will derive more contextual effects from (10) than from (9); that is, the main relevance of (8) lies in the embedded proposition in (10). Since the main relevance of (8) lies in the embedded proposition, the performative can occupy a parenthetical position, as in (11).

(8) I predict that Jane will leave the room. (Blakemore 1992: 97)

(9) The speaker is predicting that Jane will leave the room.

(10) Jane will leave the room.

(11) Jane will, I predict, leave the room. (Blakemore 1992: 98)

In contrast, the main relevance of a performative describing a communicated act lies in the higher-level proposition rather than in the embedded proposition according to Blakemore, and so such performatives cannot occupy parenthetical positions, as (12) illustrates.

(12) ! Belle d'Azur will win the race, I bet \$100. (Recanati 1987, cited in Blakemore ibid.)

In summary, "parenthetical verbs should name non-communicated acts rather than communicated acts." (Blakemore 1991: 204)

Blakemore considers an apparent counterexample, where a communicated act, promising, is named by a parenthetical verb. She argues that the examples below "have much more the quality of a reassurance than a promise." However, whilst (13) clearly is a case of reassurance, since it describes an event which is beyond the speaker's control, (14) describes an act which is within the speaker's control and can therefore be interpreted as 
a promise. There is thus very little difference between (14) and the non-parenthetical (15).

(13) You'll enjoy it, I promise.

(14) I'll be there, I promise.

(15) I promise I'll be there.

Conversely, some verbs naming what, for Blakemore, are non-communicated acts are inappropriate when used parenthetically:

! Jane will, I claim, leave the room.

It would appear, then, that whether or not a speech act can be described with a parenthetical verb does not provide a reliable indication of whether it is classed as a communicated or a non-communicated act in the relevance theory literature.

Moreover, the purpose of performative verbs is less problematic once the distinction between communicated and non-communincated acts is abandoned. Blakemore proposes that performative utterances perform two acts simultaneously. In (8) and (11) the speaker is communicating both that Jane will leave the room and that she is making a prediction. The latter act conveys the information that the hearer has less than conclusive evidence for the proposition communicated by the former act; that is, the performative is only relevant in so far as it helps the hearer to understand the embedded proposition (Blakemore 1991: 207; 210f). However, as Franken (1997) notes, supposing two acts of communication entails two applications of the principle of relevance. Two applications of the principle of relevance requires two processes of inferential enrichment and two propositional forms, yet Blakemore (1991: 210) treats the acts as occurring simultaneously. If, as I have argued, the identification of speech acts such as predicting and warning constitutes an integral part of the process of comprehending utterances which convey them, then we do not need to treat performatives as special cases in which two acts occur simultaneously.

If we accept that there are no non-communicated acts (that is, speech acts whose identification is not essential to the interpretation of an utterance containing them), then we can propose a unified account of the purpose of performative expressions. In the absence of a performative verb, the identification of the speech act performed by an utterance is an inferential process, and so information such as 'The speaker is predicting $X$ ' or 'The speaker is warning that $\mathrm{X}^{\prime}$ is implicit information. It is always possible for a speaker to make implicit information explicit, though it may not always be desirable to do so (Nicolle 1999). In uttering a performative such as (8) or (11) a speaker is engaging in 'strong communication', that is, she is reducing the hearer's responsibility for the determination of contextual effects by constraining his interpretation processes. There may be various reasons why a speaker should wish to do this; she may believe that the hearer might otherwise misinterpret her utterance, or she may wish to increase the salience of a particular speech act in order to encourage the hearer to compute additional contextual effects that he might otherwise not recover. 


\section{Conclusion}

In the relevance theory literature (Sperber and Wilson 1986: 245; Blakemore 1991) a communicated speech act was defined purely from the perspective of the hearer as an act whose identification is essential to the comprehension of an utterance. In the preceding discussion I argued that speech acts which indicate either the strength with which an assumption is held or socially relevant information must be communicated acts in the relevance theory usage of the term. The arguments presented differed in each case, but were based on the claim that both types of information are inherently relevant and therefore essential to the comprehension of utterances which contain them. This claim follows from aspects of relevance theory which are more fundamental than the supposed distinction between communicated and non-communicated acts.

Computing the strength of an assumption is an essential element in the derivation of contextual effects (contextual implications, and strengthening or elimination of existing assumptions), which is the goal of utterance interpretation according to relevance theory. Since determining the strength of an assumption is essential to the comprehension of an utterance, speech acts which indicate the strength with which an assumption is held must, given the definition of a communicated act, be communicated. Speech acts which convey socially relevant information are likewise communicated acts since information pertaining to the maintenance and negotiation of power relations and other aspects of the interlocutors' social environment comes with a guarantee of optimal relevance, that is, adequate contextual effects for minimal processing effort. Such information is relevant because the structure of human society ensures that it generally yields adequate contextual effects and the structure of the human cognitive system ensures that such information is easy to process.

In both cases, since a speaker knows that a hearer is bound to attempt to identify such speech acts, it is in her interest to ensure that the hearer identifies the correct (or intended) acts. Such speech acts are also therefore communicated acts from the perspective of the speaker, that is, they are speech acts which the speaker intends the hearer to recover.

These arguments also simplify the analysis of performative utterances. If we assume that acts such as predicting and warning are always communicated, we do not need to posit the occurrence of two simultaneous acts of communication when a performative is uttered. Rather than constituting a special case, such utterances can be treated in the same way as any other declarative utterance.

Since all the supposed non-communicated acts discussed in the relevance theory literature indicate either the strength with which an assumption is held or socially relevant information, it follows that all these speech acts are in fact communicated. Rather than constituting an argument against relevance theory, this conclusion follows from some basic tenets of relevance theory and suggests that the distinction between communicated and noncommunicated acts should be abandoned.

\section{References}

Andersen, G., and T. Fretheim (in press) (eds.) Pragmatic markers and propositional attitude. Amsterdam/Philadelphia: John Benjamins Publishing Company. 
Arundale, R.B. (1999) An alternative model and ideology of communication for an alternative to politeness theory. Pragmatics 9: 119-153.

Blakemore, D. (1991) Performatives and parentheticals. Proceedings of the Aristotelian Society 91: 197-213.

Carston, R., and S. Uchida (1998) (eds.) Relevance theory: Applications and implications. Amsterdam/Philadelphia: John Benjamins Publishing Company.

Cosmides, L., and J. Tooby (1992) Cognitive adaptations for social exchange. In J. Barkow, L. Cosmides and J. Tooby (eds.), The adapted mind: Evolutionary psychology and the generation of culture. Oxford: Oxford University Press.

Dominicy, M., and N. Franken (forthcoming) Speech acts and relevance theory. To appear in D. Vanderveken and S. Kubo (eds.), Essays in speech act theory. Amsterdam/Philadelphia: John Benjamins Publishing Company.

Escandell-Vidal, V. (1996) Towards a cognitive approach to politeness. In K. Jaszczolt and K. Turner (eds.), Contrastive semantics and pragmatics. Oxford: Pergamon Press, pp. 629-650.

Franken, N. (1997) Les performatifs dans la théorie de la pertinence. Paper presented at the 3ème Rencontre des Jeunes Linguistes, Université du Littoral, Dunkerque, 16-17 May 1997.

Jary, M. (1998a) Relevance theory and the communication of politeness. Journal of Pragmatics 30: 1-19.

Jary, M. (1998b) Is relevance theory asocial? Revista Alicantina de Estudios Ingleses 11: 157-169.

Humphrey, N. (1976) The social function of the intellect. In P.P.G. Bateson and R.A. Hinde (eds.), Growing points in ethology. Cambridge: Cambridge University Press.

Myers-Scotton, C. (1993) Common and uncommon ground: Social and structural factors in codeswitching. Language in Society 22: 475-503.

Myers-Scotton, C. (1995) What do speakers want? Codeswitching as evidence of intentionality in linguistic choices. In P. Silberman and J. Loftin (eds.), SALSA 2 (Papers from the Symposium about Language and Society at Austin). Austin: University of Texas, Department of Linguistics.

Nicolle, S. (1999) On the translation of implicit information: Experimental evidence and further considerations. Notes on Translation 13.3: 1-12.

Nicolle, S. (in press) Markers of general interpretive use in Amharic and Swahili. In G. Andersen and T. Fretheim (eds.), Pragmatic markers and propositional attitude. Amsterdam/Philadelphia: John Benjamins Publishing Company, pp. 174-188.

Okamoto, S. (1999) Situated politeness: Manipulating honorific and non-honorific expressions in Japanese conversations. Pragmatics 9: 51-74.

O’Neill, J. (1988-89) Relevance and pragmatic inference. Theoretical Linguistics 15: 241-261.

Recanati, F. (1987) Meaning and force. Cambridge: Cambridge University Press.

Rouchota, V., and A. Jucker (1998) (eds.) Current issues in relevance theory. Amsterdam/Philadelphia: John Benjamins Publishing Company.

Sperber, D. (1994) The modularity of thought and the epidemiology of representations. In L.A. Hirschfeld and S.A. Gelman (eds.), Mapping the mind: Domain specificity in cognition and culture. Cambridge: Cambridge University Press, pp. 39-67. 
Sperber, D., and D. Wilson (1986) Relevance: Communication and cognition. Oxford: Basil Blackwell.

Terkourafi, M. (1999) Frames for politeness: A case study. Pragmatics 9: 97-117.

Tooby, J. and L. Cosmides (1992) The psychological foundations of culture. In J. Barkow, L. Cosmides and J. Tooby (eds.), The adapted mind: Evolutionary psychology and the generation of culture. Oxford: Oxford University Press.

Wilson, D. and D. Sperber (1993) Linguistic form and relevance. Lingua 90: 1-25. 